\title{
Definições e terminologias para Sistema Integrado de Produção Agropecuária $^{1}$
}

\author{
Definitions and terminologies for Integrated Crop-Livestock System
}

\author{
Paulo César de Faccio Carvalho²*, Anibal de Moraes $^{3}$, Laíse da Silveira Pontes ${ }^{4}$, Ibanor Anghinoni ${ }^{5}$, Reuben \\ Mark Sulc ${ }^{6}$ e Caterina Batello ${ }^{7}$
}

\begin{abstract}
RESUMO - A pesquisa sobre Sistema Integrado de Produção Agropecuária tem experimentado crescente importância no Brasil. Há incremento no número de publicações neste tópico, refletindo seu renovado interesse. No entanto, a literatura brasileira que referencia esse sistema é confusa, por causa de diferentes termos, siglas e conceitos utilizados. Muitas publicações refletem características regionais, sem aderência à terminologia internacional. Há necessidade de avançar no uso de terminologias e definições mais precisas, visando garantir clareza na comunicação nacional e internacional. O objetivo deste artigo é desenvolver consenso para esclarecer definições e terminologias a serem adotadas na descrição do Sistema Integrado de Produção Agropecuária. A regra proposta é usar a terminologia adotada pela Food and Agriculture Organization of the United Nations como referência. Espera-se que os termos e definições discutidos tornem-se padrão para uso em publicações referentes a esse sistema, e auxiliem para uniformidade de uso na língua portuguesa e de palavraschave usadas para indexação internacional. Por fim, espera-se que essa padronização ajude na apropriada comunicação em áreas como ciência, educação e indústria, a fim de promover essa tecnologia como caminho a seguir visando à intensificação sustentável na produção de alimentos.
\end{abstract}

Palavras-chave: Integração lavoura-pecuária. Sistema misto de produção. Terminologia.

\begin{abstract}
Research on Integrated Crop-livestock System has experienced growing importance in Brazil. There is an increasing number of publications on this topic, reflecting the renewed interest. However, the Brazilian literature referring to those systems is confusing, because of different terms, acronyms and concepts used. Most publications about those systems reflect regional features with no international reference. There is a need to move the language forward toward more precise and meaningful terminology and definitions, to ensure clear national and international communication. The aim of this manuscript is to develop a consensus of clear definitions and terminology for use in describing Integrated Crop-livestock System. The key rule proposed is to use the terminology adopted by the Food and Agriculture Organization of the United Nations as the main reference. It is hoped that the terms and definitions discussed become the standard for use in publication on Integrated Crop-livestock System and will provide a more uniform national language and keywords used for international citation. Finally, it is hoped that this standard will help improve communication in science, education, and industry, in order to foster this technology as a way forward toward sustainable intensification of food production.
\end{abstract}

Key words: Integrated crop-livestock system. Mixed crop-livestock system. Terminology.

\footnotetext{
*Autor para correspondência

Recebido para publicação em 13/05/2014; aprovado em 02/09/2014

Pesquisa em Agrobiodiversidade e Sustentabilidade Agropecuária/REPENSA, processo 562688/2010-2

${ }^{2}$ Departamento de Plantas Forrageiras e Agrometeorologia, Universidade Federal do Rio Grande do Sul, Av. Bento Gonçalves, 7712 , Porto Alegre-RS, 91.501-970, paulocfc@ufrgs.br

${ }^{3}$ Departamento de Fitotecnia e Fitossanitarismo, Universidade Federal do Paraná, Rua Dos Funcionários, 1.540, Curitiba-PR, Brasil, 80.035-050, anibalm@ufpr.br

${ }^{4}$ Instituto Agronômico do Paraná, Av. Euzébio de Queiroz, s/n, Caixa Postal 129, Ponta Grossa-PR, 84.001-970, laisepontes@iapar.br

${ }_{5}^{5}$ Departamento de Solos, Universidade Federal do Rio Grande do Sul, Av. Bento Gonçalves, 7712, Porto Alegre-RS, 91.501-970, ibanghi@ufrgs.br ${ }^{6}$ Horticulture and Crop Science/The Ohio State University, 2021 Coffey Rd Columbus, OH 43210, sulc.2@osu.edu

${ }^{7}$ Plant Production and Protection Division, Food and Agriculture Organization of the United Nations, Viale delle Terme di Caracalla, 00153,

Rome, Caterina.Batello@fao.org
} 


\section{INTRODUÇÃO}

O Sistema Integrado de Produção Agropecuária (SIPA) está presente em 25 milhões de $\mathrm{km}^{2}$ (BELL; MOORE, 2012) e é responsável por aproximadamente $50 \%$ da produção de alimentos no mundo - $65 \%$ dos bovinos, $75 \%$ do leite e $55 \%$ dos cordeiros nos países em desenvolvimento (HERRERO et al., 2010). Por essa representatividade, ele é considerado vital para a segurança alimentar em âmbito global. Além do seu papel na produção de alimentos, esse sistema é a base da produção dos países em desenvolvimento, onde dois bilhões de pessoas são sustentadas por esse modelo de produção (WRIGHT et al., 2011).

Recentemente, o SIPA foi reconhecido como alternativa para intensificação sustentável (FAO, 2010), pois reúne gama de atributos raros em sistemas de produção de alimentos. Ele é mais eficiente no uso dos recursos naturais (WRIGHT et al., 2011); promove ciclagem de nutrientes e melhoria do solo (SALTON et al., 2014); reduz os custos de produção (RYSCHAWY et al., 2012), mantendo níveis de produtividade elevados (BALBINOTJR et al., 2009); e ainda produz inúmeros serviços ecossistêmicos (SANDERSON et al., 2013). Resulta que tal sistema retomou sua importância, após décadas de predomínio de sistemas intensivos, os quais se caracterizam por pouca diversidade e pelo elevado uso de insumos (LEMAIRE et al., 2014). Segundo Herrero et al. (2010), o futuro da alimentação do planeta está, agora, em tecnologias de intensificação sustentável que promovam ganhos de eficiência para se produzir mais alimentos sem que se use mais área, água ou outros insumos.

NoBrasil, este fenômenode ressurgimentodoSIPA se tornou notório, recentemente, pois sendo reconhecido como promovedor de sequestro de carbono, logo foi incluído na agenda da produção agrícola mitigadora dos gases de efeito estufa (Plano de Agricultura de Baixa Emissão de Carbono, Ministério da Agricultura, Pecuária e Abastecimento). Esta notoriedade parece vir causando frêmito na comunidade técnico-científica brasileira. Como é comum em situações como esta, indivíduos e instituições procuram encontrar elementos de aderência aos novos rumos ditados pelos agentes políticos e pelas agências financiadoras. Alguns estabelecem novas linhas de pesquisa nesse tema, enquanto outros procuram adaptar a linha de pesquisa anterior às novas demandas. É quando surgem "novas terminologias e conceitos" que procuram refundar o tema ou enquadrar necessidades regionais (tipos de sistemas, de cultivos, de animais, etc.), o que provoca imprecisão e dubiedade no meio acadêmico, científico e técnico. A título ilustrativo, a seguir, quatro exemplos recentes são apresentados em ordem cronológica, assumindo-se que todos estejam referindo-se ao mesmo sistema.
Macedo (2009) apresenta uma definição consensual de integração lavoura-pecuária proposta por pesquisadores de várias unidades da Embrapa: "[...] sistemas produtivos de grãos, fibras, carne, leite, lã, e outros, realizados na mesma área, em plantio simultâneo, sequencial ou rotacionado [sic], onde se objetiva maximizar a utilização dos ciclos biológicos das plantas, animais, e seus respectivos resíduos, aproveitar efeitos residuais de corretivos e fertilizantes, minimizar e otimizar a utilização de agroquímicos, aumentar a eficiencia no uso de máquinas, equipamentos e mão-de-obra, gerar emprego e renda, melhorar as condições sociais no meio rural, diminuir impactos ao meio ambiente, visando à sustentabilidade." Em Português, o autor apresenta o acrônimo SILPs, traduzindo-o para o Inglês como CLIS, isto é, Crop-Livestock Integrated Systems.

Balbinot $\mathrm{Jr}$ et al. (2009) definem a Integração Lavoura-Pecuária (ILP) como "[...] um sistema que alterna, na mesma área, o cultivo de pastagens anuais ou perenes, destinadas à alimentação animal, e culturas destinadas à produção vegetal, sobretudo grãos [...]" (denominam Crop-Livestock System em inglês, mas não apresentam acrônimo). Já Balbino et al. (2011) apresentam a integração lavoura-pecuária-floresta sob o acrônimo iLPF (CLF em sua tradução para o inglês, referindo-se a Crop-LivestockForestry Integration), como sendo "[...] uma estratégia que visa à produção sustentável, que integra atividades agrícolas, pecuárias e florestais realizadas na mesma área, em cultivo consorciado, em sucessão ou rotacionado [sic], e busca efeitos sinérgicos entre os componentes do agroecossistema, contemplando a adequação ambiental, a valorização do homem e a viabilidade econômica." No que Balbino et al. (2011) nominaram Marco Referencial da Integração LavouraPecuária-Floresta, reportam que a iLPF contemplaria quatro modalidades de sistemas: i) integração lavoura-pecuária; ii) integração lavoura-pecuária-floresta; iii) integração pecuáriafloresta; iv) integração lavoura-floresta.

Em sua definição, Balbinoetal.(2011) ressaltam que esses sistemas se assemelham à classificação dos sistemas silviagrícola, silvipastoril e agrossilvipastoril, mas “[...] $a$ iLPF é uma estratégia que apresenta classificação mais abrangente, incluindo integração lavoura-pecuária." Já Anghinoni et al. (2013) referem-se a "[...] sistemas integrados de produção agrícola e pecuária [...]”, os quais seriam caracterizados "[...] por serem planejados para explorar sinergismos e propriedades emergentes, frutos de interações nos compartimentos solo plantaanimal-atmosfera de áreas que integram atividades de produção agrícola e pecuária." (Integrated CropLivestock systems, ICLS, acrônimo e definição em inglês descritos em Moraes et al. 2013.

Como se pode depreender, tanto os termos em português como aqueles em inglês usados para referenciar 
o sistema não são consonantes, tampouco os termos para indexação nas bases internacionais. Os acrônimos são diversos, e as definições não parecem tratar do mesmo sistema. Não obstante, todos tratam do mesmo sistema. Não deveriam, portanto, tratar o mesmo assunto de modo tão diverso, pois resulta imprecisão, o que não é apropriado para a comunicação técnico-científica.

Segundo Tanaka et al. (2008), não há como fazer pesquisa relevante em SIPA (o autor se refere a Integrated Crop-Livestock Systems) sem o claro entendimento dos termos, conceitos e objetivos filosóficos que norteiam o sistema. Fenômeno semelhante está ocorrendo com o sistema plantio direto, havendo registros de conflito no entendimento e na difusão dos resultados de pesquisa por falta de padronização quanto ao correto significado do termo (DERPSCH et al., 2014). Nesse contexto, este manuscrito objetiva auxiliar o meio técnico-científico a padronizar seu entendimento, conceito e uso de termos para a literatura nacional, bem como seu alinhamento com as terminologias internacionais, facilitando a disseminação do conhecimento com relação a esse sistema, e também a citação da pesquisa nacional nas bases internacionais de referência.

\section{BREVE HISTÓRICO DO SISTEMA INTEGRADO DE PRODUÇÃO AGROPECUÁRIA: TÃO ANTIGO QUANTO A PRÓPRIA AGRICULTURA}

É importante esclarecer que o SIPA não é novidade temática. Para contextualizar, os primeiros sistemas agrários da história humana derivam da revolução agrícola neolítica (MAZOYER; ROUDART, 2010). Ela ocorreu quando o homem caçador-coletor originou sociedades de cultivadores, fenômeno provavelmente associado ao clássico desequilíbrio entre a renovação dos recursos naturais e seu uso pelo homem (DIAMOND, 2005). Nesses primórdios da proto-agricultura, já se encontrava presente o conceito de integração dos cultivos com a exploração animal. Em seu estado bruto, o SIPA se originou, provavelmente, de uma combinação de propósitos, em que herbívoros domesticados consumiam plantas que o homem não conseguia aproveitar. Delas, os animais geravam nutrientes (e.g. carne, leite) passíveis de consumo humano, e seus excrementos geravam a fonte de nutrientes necessária aos cultivos (ANGHINONI; CARVALHO; COSTA, 2013).

Um dos primeiros registros da integração de cultivos com a produção animal data de 9000 a.C., na cidade de Jericó. Segundo van Keulen e Shiere (2004), a Bíblia (Gen. 4) sugere que Cain tinha por responsabilidade o cultivo de grãos; enquanto Abel, o cuidado dos animais. Seus desentendimentos ilustrariam, desde aquela época, as intricadas relações entre os componentes planta e animal do sistema. No Brasil, registros históricos que se referem aos sistemas agrários dos séculos XVII e XVIII já descreviam modelos de SIPA. Linhares (1995) relata um sistema tido como "peculiar e eficaz", em que o gado era integrado a cultivos de fumo e mandioca. A função do componente animal era a de estrumar o solo, no mesmo princípio da proto-agricultura.

Muito embora o conceito de integrar sistemas seja antigo, a intenção de fazê-lo de forma deliberada, diversa da simples rotação de culturas, nem tanto. $\mathrm{O}$ termo Integração Lavoura-Pecuária (ILP) foi usado nesse sentido, e explicitamente, por Medeiros (1978 apud MEDEIROS, 1973), ao sugerir o uso de bovinos de corte em áreas de cultivos de trigo e soja no Planalto do Rio Grande do Sul. Ele advogou "a coexistência da lavoura e da pecuária na mesma propriedade”, o que Moraes et al. (2014a) consideraram, no Brasil, um avanço de maturidade do SIPA como conceito distinto da simples rotação de cultivo/pastagens. Moraes et al. (2014a) avaliaram que a última evolução do SIPA, nacionalmente, foi provocada pela tecnologia do plantio direto, quando a pecuária passou a ter por desafio se adaptar a sistemas profundamente direcionados a práticas conservacionistas.

Portanto, os sistemas integrados são tão antigos quanto a domesticação das plantas e dos animais. Porém, sempre estiveram em evolução, pois o conhecimento de suas propriedades evolui com o conhecimento humano.

\section{TERMOS E DEFINIÇÕES PARA SISTEMA INTEGRADO DE PRODUÇÃO AGROPECUÁRIA}

Na premissa de que um conceito, em ciência, não possa se delimitar por entendimentos locais, e de que a comunicação científica deva contemplar linguagem comum de difusão global, propõe-se que se aborde a temática com referência à FAO (Food and Agriculture Organization of the United Nations). A FAO é um organismo das Nações Unidas cuja representatividade alcança 191 países, incluindo o Brasil. Seus documentos trazem, em tese, a concordância unânime de todos os países signatários.

Em sua definição, a FAO (2010) apresenta uma contextualização que praticamente não é citada na literatura nacional, qual seja, a possibilidade de os sistemas integrados serem compostos por áreas distintas, não somente dentro de uma mesma propriedade, mas entre diferentes propriedades e regiões (Box 1).

Box 1 - Definição de Sistema Integrado de Produção Agropecuária (FAO, 2010, http://www. fao.org/fileadmin/templates/agphome/images/iclsd/ documents/crop_livestock_proceedings.pdf)

Integration can be on-farm as well as on an areawide basis that may involve some specialization. Successful 
integration involves an intentional integration that reflects a synergistic relationship among the components (the whole is greater than the sum of the parts) of crops, livestock and/or trees and that this synergistic relationship when appropriately managed results in enhanced social (including community), economic and environmental sustainability and improves the livelihoods of those farmers who manage them.

Uma segunda constatação está na premissa de haver intenção deliberada em explorar sinergismos entre componentes, o que difere esse sistema daqueles que visam puramente à rotação de culturas com outros propósitos, ou à simples exploração mais eficiente do espaço e diversificação da renda. O sistema deve ser planejado, construído pela escolha dos componentes em seus arranjos no tempo e no espaço, objetivando sinergismos nos resultados produtivos, sociais e ambientais. As dimensões social e econômica estão incluídas na definição da FAO, compondo a exigência de sustentabilidade. Não há definição explícita dos componentes, mas contemplam-se plantas e animais.

Em suas cinco línguas oficiais, a FAO (http:// termportal.fao.org/faoterm/main/start.do) assim denomina o sistema integrado: i) Inglês: Integrated Crop-Livestock System; ii) Espanhol: Sistema Integrado de Producción Agropecuária; iii) Francês: Système de Production Intégré Culture-Élevage; iv) Chinês: 农牧综合系统; Árabe:

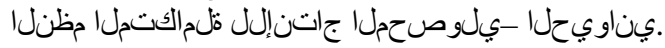

A FAO ainda utiliza um segundo termo em seus documentos: mixed cropping (Box 2). Ele parece menos empregado em documentos recentes, e mais especificamente associado a pequenos produtores na África e Ásia.

\section{Box 2 - Mixed cropping (http://termportal.fao.org/ faooa/main/start.do)}

Different mix of crops, trees, animals, fish to ensure variety of food, fodder and fiber sources and complementary use of natural resources. It also brings more ecosystem stability. Mixed cropping is a system of sowing two or three crops together on the same land, one being the main crop and the others the subsidiaries.

- Source Glossaryofstatisticalterms, Organisation for Economic Co-Operation and Development, OECD (http://stats.oecd.org/glossary/detail.asp?ID=1666); Natural Resources Management and Environment Department, NR, FAO, 2009; KCCM, FAO, 2009.

\section{Related Terms: intercropping; polyculture.}

O Português não é língua oficial na FAO, mas sua proximidade com a língua espanhola sugere o termo oficial em Espanhol traduzido para Sistema Integrado de Produção Agropecuária, adotado desde o início neste manuscrito. $\mathrm{O}$ termo em Português sugere o acrônimo SIPA, enquanto em
Inglês sugere ICLS, como de fato é usado em inúmeros artigos recentes (e.g. FRANZLUEBBERS; STUEDEMANN, 2013; PIVA et al., 2013; SALTON et al., 2013).

A denominação Integração Lavoura-Pecuária é a mais comumente encontrada na literatura nacional (vide MORAES et al., 2014b, neste número; consulte-o também como ilustração das dificuldades no rastreamento dos termos indexados na literatura). Não obstante, Integração Lavoura-Pecuária não é termo tecnicamente correto (ver Box 3), pois não abrange as tipologias que podem ser enquadradas na terminologia empregada pela FAO.

\section{Box 3 - Definições dos termos envolvidos segundo o} Dicionário de Português Aurélio (FERREIRA, 2009)

lavoura. [Dev. do ant. lavorar < tal. laborare, "trabalhar".] S.f. 1. Preparação do terreno para a sementeira ou plantação; lavra. 2. Amanho e/ou cultivo da terra; lavra, lavradio, lavragem, lavramento; agricultura. [Sin. (angol.) nesta acepç.: tonga] 3. Terreno lavrado e cultivado; lavra, lavrada. 4. Bras. Trabalho habitual ou profissão. [var.: lavoira.]

pecuária. [F. subst. do adj. pecuário.] S.f. Arte e indústria do tratamento e criação do gado.

agropecuária. [De agro - + pecuária.] S.f. Teoria e prática da agricultura e da pecuária, nas suas relações mútuas.

Em âmbito mundial, o componente animal abrange tipos de animais incomuns aos modelos brasileiros, como o uso de peixes, patos, suínos, frangos, caprinos, perus, coelhos, equinos, avestruz, lhamas, ovinos, búfalos e outros (BONAUDO et al., 2013; DEVENDRA; DOVE; HILIMIRE, 2011; KIRKEGAARD, 2014; THOMAS, 2002; WRIGHT et al., 2011), incluindo contribuições outras que não somente a produção animal, como tração e controle de pragas. Nos sistemas africanos e asiáticos, o componente animal mais comumente interage alimentando-se dos resíduos das culturas, enquanto no Brasil o sistema está associado com a rotação de pastagens e ruminantes em pastejo direto, muitas vezes conexo com a recuperação de pastagens degradadas (CARVALHO; MORAES, 2013; MACEDO, 2009; MORAES et al., 2014a). Mundialmente, o componente vegetal inclui os mais diversos tipos de cultivos de grãos, fibras, frutas, madeira, óleo, etc., incluindo pastejo direto, em rotação ou em cultivo associado, árvores incluindo frutíferas, hortigranjeiros e outros (DEVENDRA; HILIMIRE, 2011; SPRAGUE et al., 2014; THOMAS, 2002).

Portanto, o termo Integração Lavoura-Pecuária, empregado mais comumente na literatura brasileira, não abrange a diversidade de tipologias que os sistemas integrados podem alcançar. Seu emprego se assemelha ao do Sistema 
Plantio Direto, que também não é correto tecnicamente. Mas, assim como este exemplo, uma vez sendo de amplo domínio e consagrado, o termo acaba por se consolidar. Contudo, seu emprego deveria ser tomado como termo coloquial, como nome comum em relação a um nome científico, em literatura voltada mais ao público técnico que ao científico.

Um outro elemento, recente, a considerar na literatura brasileira é a inclusão do componente florestal no termo Integração Lavoura-Pecuária, originando a Integração Lavoura-Pecuária-Floresta (ILPF ou iLPF, dependendo da citação de origem). Como apresentado anteriormente, o termo incluiria quatro diferentes tipos de sistemas, dando margem aos acrônimos ILPF, ILP, IPF e ILF, segundo a combinação dos componentes do sistema. Assim como pontuado por Balbino et al. (2011), os termos se assemelham à classificação de sistemas agroflorestais (mas se poderia dizer que se confundem), para o que novamente se deva referir a FAO (Box 4 e 5).

\section{Box 4 - Sistemas agroflorestais e silvipastoris}

\section{Agroforestry (http://termportal.fao.org/faooa/main/ start.do)}

Agroforestry is a collective name for land-use systems and technologies where woody perennials (trees, shrubs, palms, bamboos, etc.) are deliberately used on the same land management unit as agricultural crops and/or animals, in some form of spatial arrangement or temporal sequence. In agroforestry systems there are both ecological and economic interactions between the different components.

Source Terminology for integrated resource planning and management, 1999 - X2079E; Natural Resources Management and Environment Department, NR, FAO, 2009; KCCM, FAO, 2009.

Related Terms: silvopastural practice; agrosilviculture; silviculture; silvopastural practice; silvipasture.

\section{Box 5 - Sistemas silvipastoris}

\section{Silvopastoral practice (http://termportal.fao.org/ faooa/main/start.do)}

A form of agroforestry that combines forestry and grazing for animals. In certain areas, silvopastoral practices can offer an alternative to cattle production systems based solely on pasture. Such practices include planting high densities of trees and shrubs in pastures, cut-and-carry systems whereby livestock are fed with the foliage of specifically planted trees and shrubs in areas previously used for other agricultural practices, and using fast-growing trees and shrubs forfencing and wind screens. The on-site benefits of silvopastoral practices to land users include additional production from the tree component, such as fruit, fuelwood, fodder or timber; maintaining or improving pasture productivity by increasing nutrient recycling; and diversification of production. Silvopastoral practices also have important biodiversity benefits. They have been shown to play a major role in the survival of wildlife species by providing scarce resources and refuge; to have a higher propagation rate of native forest plants; and to provide shelter for wild birds. They can also help connect protected areas. In addition, silvopastoral practices can fix significant amounts of carbon in the soil and in the standing tree biomass and have a beneficial effect on water services.

- Source SOFA, FAO 2007 (ftp://ftp.fao.org/ docrep/fao/010/a1200e/a1200e00.pdf); Natural Resources Management and Environment Department, NR, FAO, 2009; KCCM, FAO, 2009.

Como se pode depreender, os sistemas agroflorestais não preveem integração entre áreas distintas, tampouco abrangem as tipologias de integração dos componentes planta e animal que existem no SIPA. Além disso, tais sistemas não têm, em sua definição, o propósito de explorar sinergismos como, por exemplo, via ciclagem de nutrientes, que se constitui num dos pilares conceituais do SIPA (ANGHINONI; CARVALHO; COSTA, 2013).

Outro fator a considerar é que o termo ILPF e demais acrônimos combinantes sugerem a compartimentalização dos componentes envolvidos, o que fere o princípio filosófico-sistêmico próprio dos sistemas integrados (GRIFFON, 2013). Ademais, denotam ausência de visão holística e carecem de aderência às terminologias internacionais. SendootermoILPjáincorretotecnicamente, qualquer adendo a esse acrônimo só acentua o equívoco, pois ILP é um termo coloquial.

$\mathrm{O}$ exemplo da inclusão da letra $\mathrm{F}$ para ressaltar o componente florestal, que já estava contemplado no termo coloquial "lavoura", dá margem a conceber outros casos em que hipoteticamente se queira enfatizar um dos componentes, como, por exemplo, a produção de suínos (ILPS); ou um sistema agrícola, como a produção na várzea para sistemas com arroz irrigado (ILPV); ou a particularidade que futuramente se cogite querer realçar. Como se pode concluir, este cenário deve ser evitado, para o bem da comunicação científica e da tarefa acadêmica.

\section{CONCLUSÕES}

Ao discutirem tipologias para sistemas integrados, Keulen e Schiere (2004) escreveram: “[...] it shows that much of the excitement created around new terminology derives from the presentation of old wine in new bottles." 
1. Sugere-se que na literatura técnica se use o termo Integração Lavoura-Pecuária; e na literatura científica, o termo Sistema Integrado de Produção Agropecuária. Os acrônimos em português seriam ILP e SIPA, respectivamente;

2. Para fins de indexação internacional, sugere-se o termo Integrated Crop-Livestock System e uso do acrônimo ICLS;

3. Qualquer que seja o termo utilizado, e quando oportuno, recomenda-se descrever quais components fazem parte do sistema, e como eles se arranjam nas dimensões tempo e espaço.

\section{AGRADECIMENTOS}

Este trabalho foi auxiliado por bolsa de produtividade em pesquisa do $\mathrm{CNPq}$, e pelo Projeto de Redes Nacionais de Pesquisa em Agrobiodiversidade e Sustentabilidade Agropecuária (REPENSA), Processo 562688/2010-2.

\section{REFERÊNCIAS}

ANGHINONI, I.; CARVALHO, P. C. F.; COSTA, S. E. V. G. A. Tópicos em Ciência do Solo. In: Araújo, A. P.; Avelar, B. J. R., (Eds.) Abordagem sistêmica do solo em sistemas integrados de produção agrícola e pecuária no subtrópico brasileiro... 8 . ed. Viçosa: UFV, 2013. cap. 8, p. 221-278.

BALBINO, L. C.; BARCELlOS, A. O.; STONE, L. F. Marco referencial: integração lavoura-pecuária-floresta. 1. ed. Brasília, DF: Embrapa, 2011. 130 p.

BALBINOT Jr., A. A. et al. Integração lavoura-pecuária: intensificação de uso de áreas agrícolas. Ciência Rural, v. 39, p. 1925-1933, 2009

BELL, L. W.; MOORE, A. D. Integrated crop-livestock systems in Australian agriculture: Trends, drivers and implications. Agricultural Systems, v. 111, p. 1-12, 2012.

BONAUDO, T. et al. Agroecological principles for the redesign of integrated crop-livestock systems. European Journal of Agronomy, v.57, p.43-51, 2014.

CARVALHO, P. C. F.; MORAES, A. Integration of Grasslands within Crop Systems in South America. In: LEMAIRE, G.; HODGSON, J.; CHABBI, A., (Eds.) Grasslands Productivity and Ecosystems Services. Oxfordshire: CABI, 2011. p. 219-226.

DERPSCH, R. et al. Why do we need to standardize no-tillage research? Soil \& Tillage Research, v. 137, p. 16-22, 2014.

DEVENDRA, C.; THOMAS, D. Crop-animal interactions in mixed farming systems in Asia. Agricultural Systems, v. 71, p. 27-40, 2002.
DIAMOND, J. Colapso: Como as sociedades escolhem o fracasso ou o sucesso. 2. ed. Rio de Janeiro: Record, 2005. 685 p.

DOVE, H.; KIRKEGAARD, J. Using dual-purpose crops in sheep-grazing systems. Journal of the science of food and agriculture, v. 94, n. 7, p. 1276-1283, 2014.

FAO. An international consultation on integrated croplivestock systems for development: The way forward for sustainable production intensification. Integrated Crop Management, v. 13, 64p., 2010.

FERREIRA, A. B. H. Novo dicionário Aurélio da língua portuguesa. Ferreira, M. B.; Anjos, M. dos. (Coord.) 4. ed. Curitiba: Positivo, 2009. 2120 p.

FRANZLUEBbERS, A. J.; STUEDEMANN, J. A. Soilprofile distribution of inorganic $\mathrm{N}$ during 6 years of integrated crop-livestock management. Soil and Tillage Research, v. 134, p. 83-89, 2013.

GRIFFON, M. Qu'est-ce que l'agriculture écologiquement intensive? Éditions Quæ, 2013. p. 24-25.

HERRERO, M. et al. Smart investments in sustainable food production: revisiting mixed crop-livestock systems. Science, v. 327, p. $822-825,2010$.

HILLMIRE, K. Integrated crop/livestock agriculture in the United States: A review. Journal of Sustainable Agriculture, v. 35, p. 376-393, 2011.

KEULEN, H.; SCHIERE, H. 2004. Crop-livestock systems: old wine in new bottles? In: Fischer, T. et al. (Eds.). New directions for a diverse planet. Proceedings of the IV International Crop Science Congress, Australia, 2004. 1 CD ROM.

LINHARES, M. Y. L. Religião e história agrária. Estudos Históricos, v. 15, p. 17-26, 1995.

LEMAIRE, G. et al. Integrated crop-livestock systems: Strategies to achieve synergy between agricultural production and environmental quality. Agriculture, Ecosystems \& Environment, v.190, p.4-8, 2014.

MACEDO, M. C. M. Integração lavoura e pecuária: o estado da arte e inovações tecnológicas. Revista Brasileira de Zootecnia, v. 38, p. 133-146, 2009.

MAZOYER, M.; ROUDART, L. A história das agriculturas no mundo: Do Neolítico à crise contemporânea. São Paulo: UNESP, 2010. 568 p.

MEDEIROS, R. B. Considerações sobre a integração lavoura-pecuária no Rio Grande do Sul. In: V Simpósio sobre o Manejo da Pastagem, Piracicaba. Anais... Piracicaba: ESALQ, 1978. p. 235-301.

MORAES, A. et al. Integrated crop-livestock systems in the Brazilian subtropics. European Journal of Agronomy, v. 57, p. 4-9, 2014.

MORAES, A. et al. Sistemas de integração lavourapecuária. In: REIS, R.A. et al., Eds. Forragicultura: Ciência, Tecnologia e Gestão dos Recursos Forrageiros. 1.ed. Jaboticabal, Gráfica Multipress, 2014a. p.203-218. 
MORAES, A. et al. Research on Integrated Crop-Livestock Systems in Brazil: A viewpoint. Revista Ciência Agronômica, 2014b (neste número).

PIVA, J. T. et al. Soil gaseous $\mathrm{N}_{2} \mathrm{O}$ and $\mathrm{CH}_{4}$ emissions and carbon pool due to integrated crop-livestock in a subtropical Ferralsol. Agriculture, Ecosystems \& Environment, v. 190, p. 87-93, 2014.

RYSCHAWY, J. et al. Mixed crop-livestock systems: An economic and environmental-friendly way of farming? Animal, v. 6, p. 1722-1730, 2012.

SALTON, J. C. et al. Integrated crop-livestock system in tropical Brazil: Toward a sustainable production system. Agriculture, Ecosystems \& Environment, v. 190, p. 70-79, 2014.
SANDERSON, M. A. et al. Diversification and ecosystem services for conservation agriculture: Outcomes from pastures and integrated crop-livestock systems. Renewable Agriculture and Food Systems, v. 28, p. 129-144, 2013.

SPRAGUE, S. J. et al. Crop and livestock production for dual-purpose winter canola (Brassica napus) in the highrainfall zone of south-eastern Australia. Field Crops Research, v. 156, p. 30-39, 2014.

TANAKA; D. L.; KARN, J. F.; SCHOLLJEGERDES, E. J. Integrated crop/livestock systems research: Practical research considerations. Renewable Agriculture and Food Systems, v. 23, p. 80-86, 2008.

WRIGHT, I. A. et al. Integrating crops and livestock in subtropical agricultural systems. Journal of the Science of Food and Agriculture, v. 92, p. 1010-1015, 2012. 